\title{
Down-regulation of ALKBH2 increases cisplatin sensitivity in H1299 lung cancer cells
}

\author{
Shuang-shuang WU, Wei XU, Shan LIU, Bo CHEN, Xue-li WANG, Yan WANG, Shi-feng LIU, Jian-qing WU* \\ Department of Geriatrics, the First Affiliated Hospital of Nanjing Medical University, Nanjing 210029, China
}

\begin{abstract}
Aim: To elucidate the combined effect of alkylated DNA repair protein alkB homolog 2 (ALKBH2)-targeting gene therapy and cisplatin (cDDP) chemotherapy on the non-small cell lung cancer (NSCLC) H1299 cell line.

Methods: ALKBH2 was down-regulated in $\mathrm{H} 1299$ cells by lentivirus-mediated RNA interference (RNAi). Changes in ALKBH2 expression were determined using real-time RT-PCR and Western blotting. Cell viability was evaluated using MTT assay. DNA synthesis in proliferating cells was determined using BrdU incorporation assay. Cell apoptosis was determined using flow cytometry.

Results: Lentivirus-mediated ALKBH2 silencing alone did not induce apoptosis or attenuate the growth potential of H1299 cells within five days post-infection. Combined treatment modalities with lentivirus-mediated ALKBH2 down-regulation and cDDP (333 $\mu$ mol/L) were significantly more potent in inhibiting cell growth and inducing apoptosis than mono-chemotherapy.

Conclusion: Combined treatment modalities of $\mathrm{ALKBH} 2$ knockdown and cDDP chemotherapy have the potential to improve the efficacy in the treatment of NSCLC.
\end{abstract}

Keywords: lentivirus; RNA interference; alkB homolog 2; cisplatin; non-small cell lung cancer

Acta Pharmacologica Sinica (2011) 32: 393-398; doi: 10.1038/aps.2010.216; published online 31 Jan 2011

\section{Introduction}

Lung cancer is currently the leading cause of cancer-related mortality in both men and women $(31 \%$ and $26 \%$, respectively) worldwide ${ }^{[1]}$. Four of five lung cancers are classified as non-small cell lung cancers (NSCLC), and the majority of these patients have advanced disease (stage III/IV) when diagnosed, with poor prognosis ${ }^{[2]}$. The lung cancer cure rates have not improved for 40 years, and further substantial increase in cure rates is not to be expected with the current treatment modalities. In an effort to improve survival, new therapeutic approaches focusing on the molecular mechanisms that mediate tumor cell growth or survival have gained much attention.

Combination therapy that includes a platinum-based compound has been the primary treatment for cancer patients diagnosed with advanced NSCLC ${ }^{[3]}$. Cisplatin, cis-platinum, or cis-diamminedichloroplatinum (II) (cDDP), which is classified as an alkylating agent, is a platinum-based chemotherapy drug commonly used to treat various types of cancers ${ }^{[4]}$. As the first member of the platinum complexes that now also includes carboplatin and oxaliplatin, cDDP reacts in vivo, binding to and inducing DNA cross-linking, which ultimately

\footnotetext{
* To whom correspondence should be addressed.

E-mail jianqingwu@yeah.net

Received 2010-06-10 Accepted 2010-12-02
}

triggers apoptosis. However, acquired resistance confines the application of cDDP in chemotherapeutics. Drug resistance is shown to be a cumulative process that eventually can overcome tumor regression, leading to treatment failure ${ }^{[5]}$. The lung tumor initially responds to CDDP by sensing DNA damage, which results in cell cycle arrest and apoptosis, leading to a significant reduction in tumor burden. Prolonged cDDP treatment, however, promotes the emergence and clonal expansion of drug-resistant tumor cells with enhanced repair capacity. These cDDP-resistant tumors are cross-resistant to platinum analogs, exhibit advanced histopathology, and display abundant genomic alterations ${ }^{[6]}$.

The AlkB family of dioxygenases, which directly reverse DNA base damage by oxidative demethylation, belong to the superfamily of 2-oxoglutarate- and iron (II)-dependent oxygenases $^{[7]}$. E coli AlkB is a direct dealkylation DNA repair protein that repairs single-stranded DNA (ssDNA) lesions or damaged bases in RNA using a unique oxidative dealkylation mechanism ${ }^{[8,9]}$. There are nine different potential human homologues of AlkB, denoted ALKBH1-ALKBH9 ${ }^{[10,11]}$. Two of these, ALKBH2 and ALKBH3, have similar repair activities to $E$ coli $\mathrm{AlkB}{ }^{[12-14]}$. ALKBH2 is a housekeeping enzyme and has the primary role of guarding mammalian genomes against 1-meA damage by repairing this lesion in double-stranded DNA (dsDNA). The in vivo function of ALKBH3 is still 
unclear, although it exhibits a distinct preference for repairing ssDNA and RNA ${ }^{[13]}$.

Because ALKBH2 is a direct dealkylation DNA repair protein, we hypothesized that down-regulation of ALKBH2 expression should be a promising approach to enhancing the efficacy of alkylating agents such as cisplatin, which are widely used in cancer therapy and exert anticancer effects by creating cytotoxic DNA lesions in tumor cells. In this study, we inhibited ALKBH2 expression by lentivirus-mediated RNA interference to elucidate the combined effect of ALKBH2-targeting gene therapy and cDDP chemotherapy on NSCLC cell line H1299. Here we establish the impact of lentivirus-mediated transfer of small interfering RNAs targeting ALKBH2 on human H1299 cells and demonstrate the additive efficacy of ALKBH2 knockdown combined with cDDP chemotherapy in causing tumor cell death.

\section{Materials and methods Cell culture}

The NSCLC cell line H1299 was purchased from ATCC (Manassas, VA, USA). It was maintained in Dulbecco's modified Eagle's medium (DMEM) containing 10\% fetal bovine serum (FBS) and cultured in a humidified atmosphere of $95 \%$ air and $5 \% \mathrm{CO}_{2}$ at $37^{\circ} \mathrm{C}$.

\section{Reagents and dosage}

cDDP and paclitaxel (PTX) were purchased from Sigma Chemical Co (St Louis, MO, USA). cDDP and PTX treatment was administered to cells at the concentration of $333 \mu \mathrm{mol} / \mathrm{L}$ and $100 \mathrm{nmol} / \mathrm{L}$, respectively. MTT was purchased from Shanghai Dingguo Biotechnology Co, Ltd (Shanghai, China). Annexin V-FITC Apoptosis Detection Kit was purchased from Abcam (Cambridge, MA, USA).

\section{Construction of lentiviral vector and cell infection}

A 19 nt ALKBH2-targeting sequence of oligonucleotides was designed by Oligoengine Inc (Seattle, WA, USA) and selected as the template: 5'-CCTTCAACTTTGTGCTCAT-3'. The negative construct (NC), a scrambled sequence with no homology to any human gene, was 5'-AATGTACTGCGCGTGGAGA-3'. The oligonucleotides were cloned into pGCSILGFP (GeneChem, Shanghai, China) to generate the lentiviral vectors. Recombinant lentiviral vectors and packaging vectors were then transfected into 293T cells. Supernatants containing lentiviruses expressing ALKBH2 short hairpin RNA (shRNA) or negative control shRNA were harvested $72 \mathrm{~h}$ after transfection. Then, the lentiviruses were purified using ultracentrifugation, and the titer of lentiviruses was determined. H1299 cells were infected with the lentivirus constructs at multiplicity of infection $(\mathrm{MOI})=10$, and mock-infected H1299 cells were used as negative controls.

\section{RNA extraction and quantitative real-time RT-PCR}

Total RNA was extracted from cells with Trizol reagent (Invitrogen, Carlsbad, CA, USA) according to the manufacturer's instructions. Gene expression was detected by quantitative real-time RT-PCR (qRT-PCR) using the standard SYBR Green RT-PCR kit (Takara, Otsu, Japan) according to the manufacturer's instructions. Briefly, the cDNA was synthesized using the RevertAid First-Strand cDNA Synthesis kit (MBI Fermentas, Vilnius, Lithuania) according to the manufacturer's protocol. Specific primer pairs and the amplified products are shown in Table 1. The relative levels of gene mRNA transcripts were normalized to the control $\beta$-actin. Relative gene expression was quantified using GraphPad Prism 4.0 software (GraphPad Software, San Diego, CA, USA).

Table 1. The specific primer pairs and the amplified products in quantitative real-time RT-PCR.

\begin{tabular}{|c|c|c|}
\hline Gene & Primer pairs & Product \\
\hline$A L K B H 2$ & $\begin{array}{l}\text { Sense: 5'-GACTGGACAGACCTTCAAC-3'; } \\
\text { Antisense: 5'-AGGAGACAGAGGCAATGG-3' }\end{array}$ & $122 \mathrm{bp}$ \\
\hline$B c 1-2$ & $\begin{array}{l}\text { Sense: 5'-GTGTGGAGAGCGTCAACC-3'; } \\
\text { Antisense: 5'-CTTCAGAGACAGCCAGGAG-3' }\end{array}$ & $182 \mathrm{bp}$ \\
\hline Bax & $\begin{array}{l}\text { Sense: 5'-ATGCGTCCACCAAGAAGC-3'; } \\
\text { Antisense: 5'-ACG GCG GCA ATCATCCTC-3' }\end{array}$ & $91 \mathrm{bp}$ \\
\hline$b F G F$ & $\begin{array}{l}\text { Sense: 5'-ATCAAAGGAGTGTGTGCTAACC-3'; } \\
\text { Antisense: 5'-ACTGCCCAGTTCGTTTCAGTG-3' }\end{array}$ & $178 \mathrm{bp}$ \\
\hline PDGFA & $\begin{array}{l}\text { Sense: 5'-CGGAGGAAGAGAAGCATCG-3'; } \\
\text { Antisense: 5'-GGCTGGCACTTGACACTG-3' }\end{array}$ & $179 \mathrm{bp}$ \\
\hline PDGFB & $\begin{array}{l}\text { Sense: 5'-GCACCAACGCCAACTTCC-3'; } \\
\text { Antisense: 5'-GCTTCTTCCGCACAATCTCG-3' }\end{array}$ & $150 \mathrm{bp}$ \\
\hline$\beta$-actin & $\begin{array}{l}\text { Sense: 5'-GGCGGCACCACCATGTACCCT-3'; } \\
\text { Antisense: 5'-AGGGGCCGGACTCGTCATACT-3' }\end{array}$ & 202 bp \\
\hline
\end{tabular}

\section{Western blot analysis}

The cells were lysed in lysis buffer (0.1\% SDS, 1\% NP-40, 50 mmol/L HEPES, pH 7.4, 2 mmol/L EDTA, 100 mmol/L NaCl, $5 \mathrm{mmol} / \mathrm{L}$ sodium orthovanadate, and 1\% protease inhibitor mixture set I; Calbiochem, San Diego, CA, USA) on ice for $30 \mathrm{~min}$, and the cell lysates were cleared by centrifugation at 12000 round per minute for $15 \mathrm{~min}$. Proteins were separated in $10 \%$ SDS-PAGE and electroblotted onto polyvinylidene difluoride membrane, blocked for $1.5 \mathrm{~h}$ at room temperature in 5\% BSA, and probed with anti-ALKBH2 (Cell Signaling Technology, Beverly, MA, USA), and anti-GAPDH (Sigma, St Louis, MO, USA) antibody. Following incubation with the appropriate peroxidase-conjugated secondary antibodies, chemiluminescent detection was performed with the ECL kit (Pierce Chemical Co, Rockford, IL, USA).

\section{MTT assay}

Cell viability was evaluated by MTT assay with some modifications as reported previously ${ }^{[15]}$. The test cells in exponential growth were plated at a final concentration of $2 \times 10^{3}$ cells/well in 96-well culture plates for different culture times. Ten microlitres of $10 \mathrm{mg} / \mathrm{mL}$ MTT was then added. After an additional $4 \mathrm{~h}$ of incubation, the reaction was terminated by 
removal of the supernatant and the addition of $150 \mu \mathrm{L}$ DMSO for $30 \mathrm{~min}$. Optical density (OD) of each well was measured at $570 \mathrm{~nm}$ using an ELISA reader (ELx808, Bio-Tek Instruments, Winooski, VT, USA).

\section{BrdU incorporation assay}

DNA synthesis in proliferating cells was determined using a BrdU incorporation assay ${ }^{[15]}$. After incubation with BrdU (10 $\mu \mathrm{mol} / \mathrm{L}$ ) (BD Pharmingen, San Diego, CA, USA) for $24 \mathrm{~h}$, the cells were fixed and labeled with peroxidase-conjugated antiBrdU antibody. Peroxidase substrate (tetramethylbenzidine) was then added and incubated for $30 \mathrm{~min}$; then, the absorbance values were measured at $490 \mathrm{~nm}$ using an ELISA reader (ELx808, Bio-Tek Instruments, Winooski, VT, USA). Background BrdU immunofluorescence was determined in cells not exposed to BrdU but stained with the BrdU antibody.

\section{Detection of apoptosis by flow cytometry}

Cells were simultaneously stained with fluorescein isothiocyanate (FITC) labeled annexin-V and propidium iodide (PI) to discriminate intact cells (annexin ${ }^{-} / \mathrm{PI}^{-}$) from apoptotic cells (annexin ${ }^{+} / \mathrm{PI}^{-}$) and necrotic cells (annexin ${ }^{+} / \mathrm{PI}^{+}$). A total of $1.0 \times 10^{6}$ cells were washed twice with ice-cold PBS and incubated for $30 \mathrm{~min}$ in binding buffer $(1 \mu \mathrm{g} / \mathrm{mL}$ PI and $1 \mu \mathrm{g} / \mathrm{mL}$ FITC labeled annexin-V). FACS analysis for annexin-V and PI staining was performed by an Epics Altra flow cytometer (Beckmann Coulter, Krefeld, Germany). All experiments were performed in triplicate.

\section{Statistical analysis}

Data were expressed as means \pm SD. Statistical analysis was performed using SPSS software (Release 11.0, SPSS Inc, Chicago, IL, USA). The difference between two groups was analyzed by Student's $t$-test. A value of $P<0.05$ was considered statistically significant.

\section{Results \\ ALKBH2 expression after treatment with lentivirus-mediated RNAi}

To determine the effect of RNAi on the expression of ALKBH2 in $\mathrm{H} 1299$ cells, we generated lentiviral vectors that expressed the ALKBH2 specific-RNAi or the negative control-RNAi. Three days after lentivirus infection, H1299 cells were visualized using a fluorescence microscope. The bright-field and fluorescent images showed that most cells had green fluorescent signals (Figure 1A). After the cells were infected with ALKBH2 specific-RNAi or control-RNAi, the mRNA and protein levels of ALKBH2 were analyzed. The level of ALKBH2 mRNA in cells expressing ALKBH2 specific-RNAi significantly decreased by approximately $70 \%(P<0.05)$ compared to the control cells (Figure 1B). In accordance with the decreased mRNA expression, ALKBH2 protein was also down-regulated in cells expressing the ALKBH2-specific RNAi (Figure 1C). Thus, ALKBH2-specific RNAi down-regulated ALKBH2 expression efficiently.
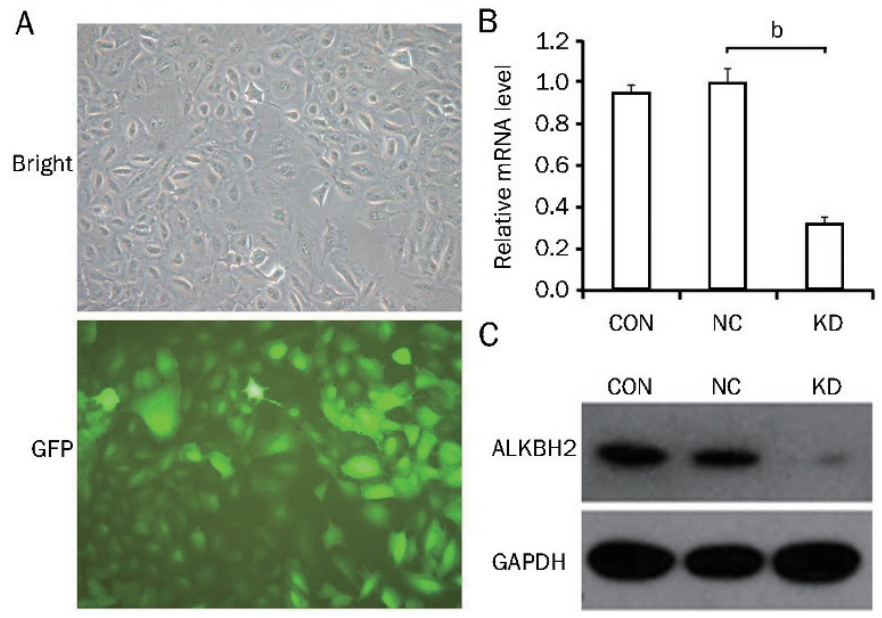

Figure 1. Down-regulation of ALKBH2 expression using lentivirusmediated RNAi. (A) Expression of lentivirus-mediated RNAi in H1299 cells. Three days after infection with lentivirus, H1299 cells were visualized using a fluorescence microscope. The phase contrast (Bright) and fluorescence (GFP) photographs were taken with the same exposure times at a magnification. (B) The relative levels of $A L K B H 2$ mRNA transcripts were analyzed by qRT-PCR. Data shown are the mean results $\pm S D$ repeated three times with triplicate samples per treatment $\left(n=3,{ }^{b} P<0.05\right)$. (C) Silencing effects of ALKBH2 protein was measured using Western blot. CON: parent cells; NC: cells infected with negative control RNAi; KD: cells infected with ALKBH2 specific RNAi.

\section{Effects of concurrent treatments of H1299 cells with lentiviruses expressing ALKBH2-specific RNAi and cDDP}

First, the MTT assay was used to investigate the potential effects of RNAi-mediated ALKBH2 down-regulation on the growth of H1299 cells. Following a 5-d incubation period, the growth of cells expressing ALKBH2-specific-RNAi was almost indistinguishable from that of the control cells (Figure 2A). We concluded that the down-regulation of ALKBH2 expression alone had no obvious impact on the growth or viability of H1299 cells. We then investigated the combined effects of ALKBH2 inhibition and cDDP treatment on H1299 cells. Forty-eight hours after infection with lentiviruses expressing ALKBH2-specific-RNAi or control-RNAi, the infected and non-infected cells were subsequently treated with cDDP (333 $\mu \mathrm{mol} / \mathrm{L})$ for $24 \mathrm{~h}^{[16]}$. The MTT assay showed that the inhibition ratio in the cells infected with ALKBH2-RNAi lentivirus (KD) was increased about 1.8-fold and 2-fold compared to the mockinfected (NC) and non-infected $(\mathrm{CON})$ cells $(P<0.05)$, respectively. These results indicate that cDDP is cytotoxic to H1299 cells and that this combination treatment resulted in lower cell viability than treatment with cDDP alone (Figure $2 \mathrm{~B}$ ). To compare the effects of ALKBH2 inhibition on cytotoxicity by a chemotherapeutic agent that does not act by inducing DNA damage, we measured the cytotoxicity of PTX on H1299 cells by MTT assay. Following $24 \mathrm{~h}$ of PTX (100 nmol/L) treatment, the inhibition ratio was not increased significantly in ALKBH2RNAi lentivirus infected cells $(P<0.05)$ compared with that in mock-infected and non-infected cells (Figure 2C). To further investigate the combined effects of ALKBH2 inhibition and 
A

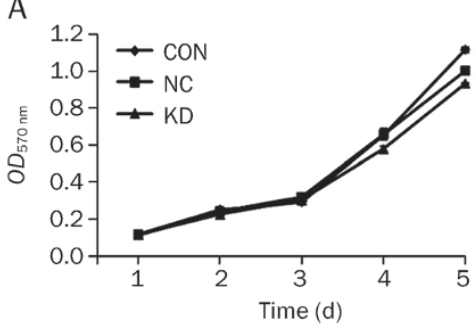

C

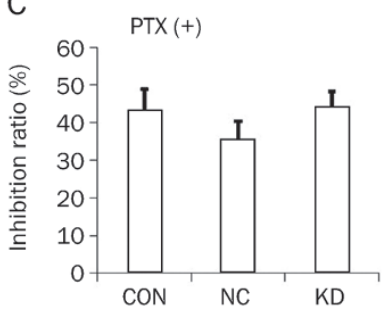

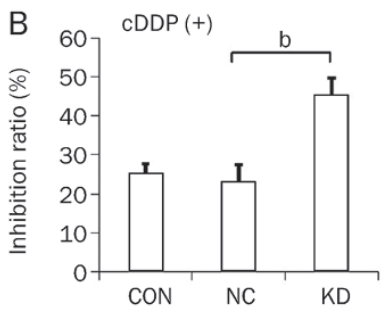

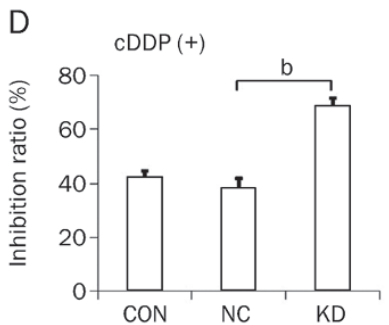

Figure 2. Effect of down-regulated ALKBH2 on $\mathrm{H} 1299$ cell growth. (A) Proliferation of parent $\mathrm{H} 1299$ cells (CON), cells infected with ALKBH2specific RNAi (KD), and cells infected with negative control RNAi (NC). Their growth dynamics were determined daily using MTT. (B) Cytotoxicity of combined cDDP-based chemotherapy with lentivirus-mediated RNAi treatment. Uninfected cells (CON), ALKBH2 RNAi infected cells or control RNAi infected cells were treated with cDDP, and the inhibition ratio was measured by MTT. Data shown are the mean results \pm SD of a representative experiment performed in triplicate $\left(n=3,{ }^{b} P<0.05\right)$. (C) Cytotoxicity of combined PTX-based chemotherapy with lentivirusmediated RNAi treatment. (D) The DNA synthesis rate in H1299 cells treated with CDDP and ALKBH2-specific RNAi was analyzed using the BrdU incorporation assay.

cDDP treatment on the DNA synthesis rate in H1299 cells, BrdU incorporation assay was performed. As shown in Figure 2D, the inhibition ratio of ALKBH2 RNAi lentivirus infected cells was enhanced significantly by treatment with cDDP $(P<0.05)$ compared to mock-infected and non-infected cells. These results indicate that concurrent treatment of H1299 cells with CDDP and RNAi targeted to ALKBH2 resulted in a lower proliferation rate compared to that of cells treated with cDDP alone.

\section{Enhancement of cDDP-induced apoptosis in $\mathrm{H} 1299$ cells by ALKBH2 RNAi}

The therapeutic efficacy of anti-cancer agents depends strongly on their ability to trigger apoptosis in target tumor cells ${ }^{[17]}$. To determine the effect of ALKBH2 knockdown on cDDPinduced apoptosis in H1299 cells, apoptosis was evaluated in uninfected cells, cells expressing ALKBH2 specific-RNAi, or negative control RNAi-expressing cells by flow cytometry analysis following cDDP treatment. As shown in Figure 3, without cDDP treatment the number of apoptotic and necrotic cells in the control, negative control and knockdown groups was almost indistinguishable. However, compared to the control cells, the addition of cDDP significantly increased apoptosis in cells infected with ALKBH2 specific-RNAi $(P<0.01)$, suggesting that combining ALKBH2 inhibition with cDDP can
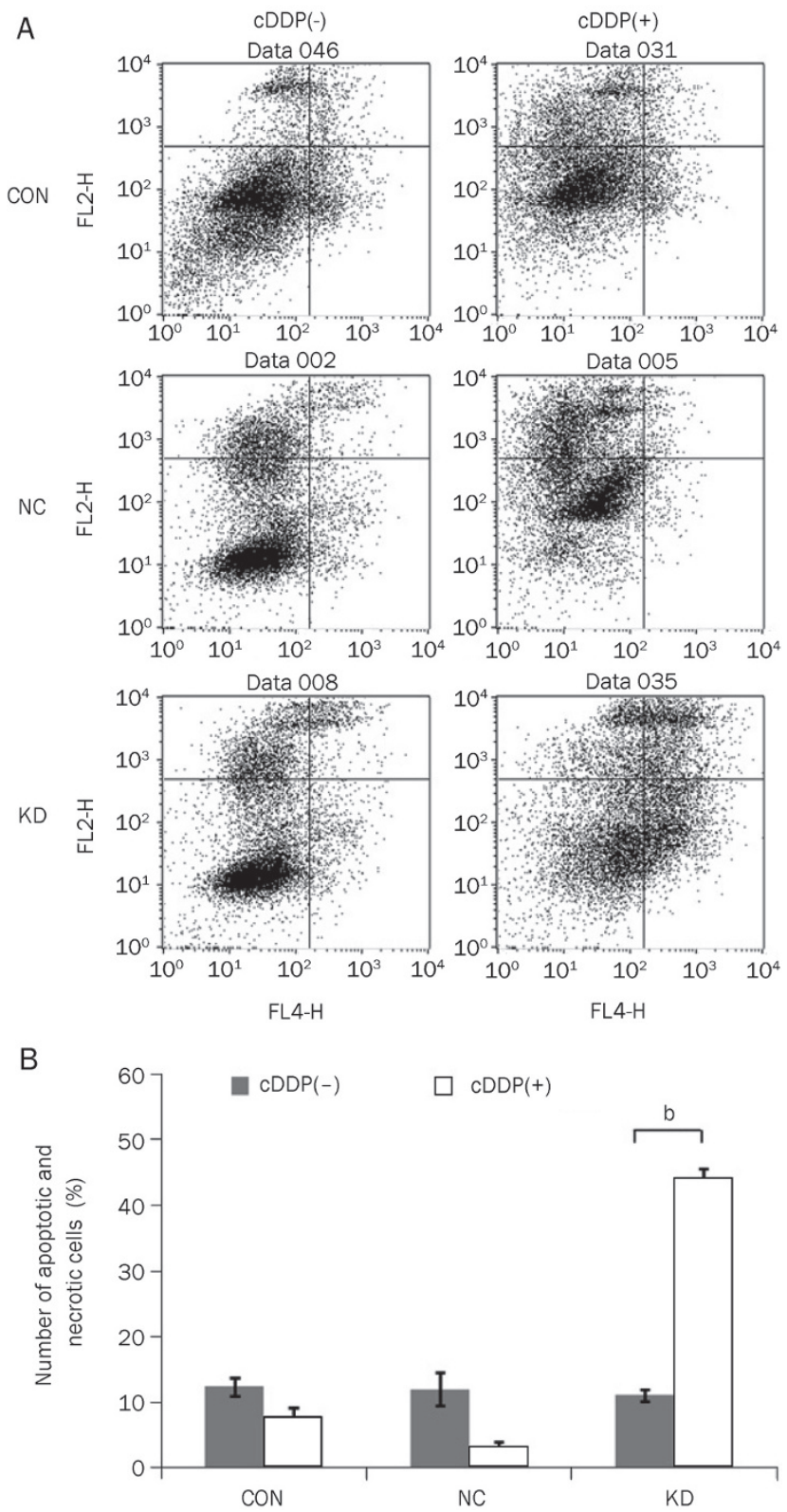

Figure 3. Enhancement of cDDP-induced apoptosis in $\mathrm{H} 1299$ cells using ALKBH2 RNAi. (A) H1299 cells were transfected with lentivirus expressing ALKBH2-specific RNAi (KD) or negative control-RNAi (NC), with the parent $\mathrm{H} 1299$ cells used as a control (CON). (B) The data presented are the average number of apoptotic and necrotic cells $( \pm S D)$ in three independent experiments. The infection with ALKBH2 RNAi (KD) enhanced the level of CDDP-induced apoptosis and necrosis compared to the negative control RNAi (NC) or the parent $\mathrm{H} 1299$ cells (CON) $(n=3$, $\left.{ }^{\mathrm{b}} \mathrm{P}<0.05\right)$.

enhance the induction of apoptosis.

Influence of ALKBH2 down-regulation on the expression of relevant genes during CDDP-induced apoptosis

Next, we investigated potential pathways involved in the enhancement of cDDP-induced apoptosis by ALKBH2 downregulation. As shown in Figure $4 \mathrm{~A}$ and $4 \mathrm{~B}$, after the addition 


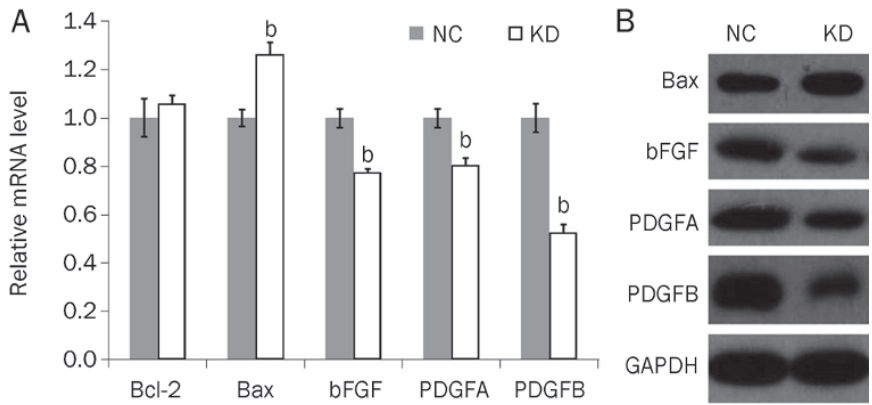

Figure 4. The effect of down-stream gene expression on the CDDPinduced apoptosis by ALKBH2 down-regulation. (A) Bax, rather than $\mathrm{Bcl}-2$ gene transcripts, was up-regulated, and bFGF, PDGFA and PDGFB genes transcripts were down-regulated in the ALKBH2 specific-RNAi infected cells using qRT-PCR. Data shown are the mean results $\pm S D$ of a representative experiment performed in triplicate $\left(n=3,{ }^{b} P<0.05\right)$. (B) Alteration of Bax, bFGF, PDGFA, and PDGFB protein expression in $\mathrm{H} 1299$ cells following $\mathrm{ALKBH} 2$ inhibition and CDDP treatment.

of cDDP, the mRNA and protein level of Bax, a member of the Bcl-2 family (Bcl-2, Bcl-XL, and Bax), was found to be up-regulated in H1299 cells infected with ALKBH2 specific-RNAi lentivirus compared to cells infected with negative control-RNAi lentivirus $(P<0.05)$. bFGF, a member of the fibroblast growth factor (FGF) family, and the platelet-derived growth factors (PDGF) PDGFA and PDGFB, were found to be down-regulated in the cells infected with ALKBH2 specific-RNAi lentivirus compared to the cells infected with negative control-RNAi lentivirus $(P<0.05)$. These results suggest that FGF and PDGF receptor tyrosine kinases signaling pathways and Bcl-2-related apoptosis pathways may be involved in the enhancement of cDDP-induced apoptosis by ALKBH2 down-regulation.

\section{Discussion}

NSCLC is the leading cause of cancer-related deaths worldwide. Unfortunately, traditional chemotherapy and radiotherapy remain inadequate for attaining disease control because of their limited efficacy, significant toxicity, development of resistance, and high relapse rates; thus, new therapeutic approaches are urgently required ${ }^{[18]}$. Combining traditional therapies with modern bioengineered therapeutics has become a new and innovative approach to the treatment of NSCLC. More recently, signal transduction pathways as well as oncogenes and tumor-suppressor genes that are known to be aberrant in lung cancer have been targeted with novel agents ${ }^{[19]}$. However, the eventual development of acquired resistance also hampers the clinical outcome of these agents during NSCLC treatment ${ }^{[20]}$. Therefore, further studies targeting multiple treatment modalities and/or the inhibition of multiple signaling pathways are required to develop a novel therapeutic agent to enhance anticancer efficacy and/or prevent treatment resistance. Intensive cancer therapy strategies have thus far focused on sensitizing cancer cells to anticancer drugmediated cell apoptosis to overcome drug resistance, and this strategy has resulted in more effective cancer therapeutics ${ }^{[21]}$. cDDP is an effective anticancer drug used to treat many types of cancer. cDDP-based combination chemotherapy is currently considered one of the most effective treatments for advanced NSCLC. The mechanism of cDDP-based anticancer activity is generally accepted to be dependent on its ability to form adducts with DNA (inter- and intra-strand DNA crosslinks) and to cause DNA strand breaks in the nucleus. These changes in the DNA interfere with normal transcription and/ or DNA replication, leading to either repair of the DNA damage and cell survival or apoptosis ${ }^{[2]}$. Some early observations have shown that cells deficient in DNA repair pathways are hypersensitive to cDDP. The AlkB family of oxygenases represents a novel class of DNA base-repair enzymes that use an oxidative dealkylation mechanism. In this study, our data show that lentivirus-mediated ALKBH2 gene silencing has no effect on basal H1299 cell growth. However, the combination of ALKBH2 RNAi lentivirus infection and cDDP-based chemotherapy is significantly more potent than cDDP monotherapy, as measured by growth inhibition and the induction of apoptosis in H1299 cells. Therefore, we hypothesized that silencing the ALKBH2 gene in CDDP-resistant patients may safely enhance the efficacy of cDDP in the treatment of lung cancer. However, the main mechanisms for ALKBH2 inhibitionmediated sensitivity to cDDP need further investigation. Our results indicate that down-regulation of ALKBH2 in cDDPtreated H1299 cells alters the expression of basic fibroblast growth factor ( $b F G F)$, platelet-derived growth factor (PDGFA, $P D G F B$ ) and Bcl-2. bFGF, an angiogenic cytokine produced by tumor cells, affects vessel formation, tumor growth, invasion and metastasis, and down-regulated bFGF may inhibit tumor growth $^{[23]}$. Cell-surface receptor tyrosine kinases play pivotal roles in different diseases, ranging from cancer to vascular disorders, so disruption of PDGF signaling pathways could inhibit tumor stromogenesis ${ }^{[24]}$. Bcl-2 family proteins regulate the propagation of apoptotic signals and cell survival and are divided into two subclasses: antiapoptotic members including Bal-2, Mcl-1, Bcl-XL, and Bcl2A1 and proapoptotic members including Bax and Bak ${ }^{[25]}$. Down-regulation of ALKBH2 in the cDDP-treated H1299 cells led to increased Bax expression and decreased $b F G F, P D G F A$, and PDGFB expression. Therefore, the regulation of these genes may be the mechanism underlying the enhanced anti-tumor effect of cDDP in combination with ALKBH2 inhibition in NSCLC therapy.

In conclusion, we demonstrate that lentivirus-mediated ALKBH2 knockdown enhances the sensitivity of human H1299 cells to cDDP-based chemotherapy. As a result of this additive efficacy of ALKBH2 gene inhibition and chemotherapy on cell death and cell proliferation in NSCLC cells, this combined treatment modality has the potential to improve the anticancer efficacy of cDDP.

\section{Acknowledgements}

This work was supported by a grant from the National Natural Science Foundation of China (№ 30971320). 


\section{Author contribution}

Shuang-shuang WU and Jian-qing WU designed the research; Shuang-shuang WU, Wei XU, Xue-li WANG, Yan WANG, and Shi-feng LIU performed the research; Shuang-shuang WU, Wei XU, Shan LIU, Bo CHEN, and Jian-qing WU analyzed the data and wrote the paper.

\section{References}

1 Jemal A, Siegel R, Ward E, Murray T, Xu J, Thun MJ. Cancer statistics, 2007. CA Cancer J Clin 2007; 57: 43-66.

2 Pfister DG, Johnson DH, Azzoli CG, Sause W, Smith TJ, Baker S Jr, et al. American Society of Clinical Oncology treatment of unresectable nonsmall cell lung cancer guideline: update 2003. J Clin Oncol 2004; 22: 330-53.

3 Ramalingam S, Belani C. Systemic chemotherapy for advanced non-small cell lung cancer: recent advances and future directions. Oncologist 2008; 13: 5-13.

4 Oliver TG, Mercer KL, Sayles LC, Burke JR, Mendus D, Lovejoy KS, et al. Chronic cisplatin treatment promotes enhanced damage repair and tumor progression in a mouse model of lung cancer. Genes Dev 2010; 24: 837-52.

5 Marcu L, Bezak E, Olver I, van Doorn T. Tumour resistance to cisplatin: a modelling approach. Phys Med Biol 2005; 50: 93-102.

6 Stordal B, Davey M. Understanding cisplatin resistance using cellular models. IUBMB Life 2007; 59: 696-9.

7 Aravind L, Koonin EV. The DNA-repair protein AlkB, EGL-9, and leprecan define new families of 2-oxoglutarate- and iron-dependent dioxygenases. Genome Biol 2001; 2: RESEARCH0007.

8 Sedgwick B. Repairing DNA-methylation damage. Nat Rev Mol Cell Biol 2004; 5: 148-57.

9 Mishina Y, He C. Oxidative dealkylation DNA repair mediated by the monomuclear non-heme iron AlkB proteins. J Inorg Biochem 2006; 100: 670-8.

10 Kurowski MA, Bhagwat AS, Papaj G, Bujnicki JM. Phylogenomic identification of five new human homologs of the DNA repair enzyme AlkB. BMC Genomics 2003; 4: 48.

11 Yang CG, Yi C, Duguid EM, Sullivan CT, Jian X, Rice PA, et al. Crystal structures of DNA/RNA repair enzymes AlkB and $A B H 2$ bound to dsDNA. Nature 2008; 452: 961-5.
12 Duncan T, Trewick SC, Koivisto P, Bates PA, Lindahl T, Sedgwick B. Reversal of DNA alkylation damage by two human dioxygenases. Proc Natl Acad Sci USA 2002; 99: 16660-5.

13 Aas PA, Otterlei M, Falnes PO, Vågbø CB, Skorpen F, Akbari M, et al. Human and bacterial oxidative demethylases repair alkylation damage in both RNA and DNA. Nature 2003; 421: 859-63.

14 Lee DH, Jin SG, Cai S, Chen Y, Pfeifer GP, O'Connor TR. Repair of methylation damage in DNA and RNA by mammalian AlkB homologues. J Biol Chem 2005; 280: 39448-59.

15 Xu Y, Wang Z, Wang J, Li J, Wang H, Yue W. Lentivirus-mediated knockdown of cyclin $Y$ (CCNY) inhibits glioma cell proliferation. Oncol Res 2010; 18: 359-64.

16 Blandino G, Levine AJ, Oren M. Mutant p53 gain of function: differential effects of different p53 mutants on resistance of cultured cells to chemotherapy. Oncogene 1999; 18: 477-85.

17 Fisher DE. Apoptosis in cancer therapy: crossing the threshold. Cell 1994; 78: 539-42.

18 Pomeroy M, Moriarty M. Clinical significance of cellular resistance in tumours to cytotoxic chemotherapy and radiotherapy. Cytotechnology 1993; 12: 385-91.

19 Murdoch D, Sager J. Will targeted therapy hold its promise? An evidence-based review. Curr Opin Oncol 2008; 20: 104-11.

20 Yang CT, Li JM, Weng HH, Li YC, Chen HC, Chen MF. Adenovirusmediated transfer of SiRNA against survivin enhances the radiosensitivity of human non-small cell lung cancer cells. Cancer Gene Therapy 2010; 17: 120-30.

21 Oh S, Kim Y, Kim J, Kwon D, Lee E. Elevated pressure, a novel cancer therapeutic tool for sensitizing cisplatin-mediated apoptosis in A549. Biochem Biophys Res Commun 2010; 399: 91-7.

22 Siddik ZH. Cisplatin: mode of cytotoxic action and molecular basis of resistance. Oncogene 2003; 22: 7265-79.

23 Bremnesa RM, Campsc C, Sirerac R. Angiogenesis in non-small cell lung cancer: The prognostic impact of neoangiogenesis and the cytokines VEGF and bFGF in tumours and blood. Lung Cancer 2006; 51: $143-58$

24 Andrae J, Gallini R, Betsholtz C. Role of platelet-derived growth factors in physiology and medicine. Gene Dev 2008; 22: 1276-312.

25 Vogler M, Dinsdale D, Dyer MJ, Cohen GM. Bcl-2 inhibitors: small molecules with a big impact on cancer therapy. Cell Death Differ 2009; 16: 360-7. 